\title{
Fault Diagnosis of Train Axle Box Bearing Based on Multifeature Parameters
}

\author{
Xiaofeng Li, ${ }^{1}$ Limin Jia, $^{2}$ and Xin Yang $^{3}$ \\ ${ }^{1}$ School of Traffic and Transportation, Beijing Jiaotong University, Beijing 100044, China \\ ${ }^{2}$ State Key Laboratory of Rail Traffic Control and Safety, Beijing Jiaotong University, Beijing 100044, China \\ ${ }^{3}$ School of Electrical Engineering, Beijing Jiaotong University, Beijing 100044, China \\ Correspondence should be addressed to Xiaofeng Li; xfengli@bjtu.edu.cn
}

Received 1 July 2014; Revised 28 August 2014; Accepted 17 September 2014

Academic Editor: Wuhong Wang

Copyright (C) 2015 Xiaofeng Li et al. This is an open access article distributed under the Creative Commons Attribution License, which permits unrestricted use, distribution, and reproduction in any medium, provided the original work is properly cited.

Failure of the train axle box bearing will cause great loss. Now, condition-based maintenance of train axle box bearing has been a research hotspot around the world. Vibration signals generated by train axle box bearing have nonlinear and nonstationary characteristics. The methods used in traditional bearing fault diagnosis do not work well with the train axle box. To solve this problem, an effective method of axle box bearing fault diagnosis based on multifeature parameters is presented in this paper. This method can be divided into three parts, namely, weak fault signal extraction, feature extraction, and fault recognition. In the first part, a db4 wavelet is employed for denoising the original signals from the vibration sensors. In the second part, five timedomain parameters, five IMF energy-torque features, and two amplitude-ratio features are extracted. The latter seven frequency domain features are calculated based on the empirical mode decomposition and envelope spectrum analysis. In the third part, a fault classifier based on BP neural network is designed for automatic fault pattern recognition. A series of tests are carried out to verify the proposed method, which show that the accuracy is above $90 \%$.

\section{Introduction}

As one of the key components of railway vehicles, the operation condition of the axle box bearing has a significant effect on traffic safety. Effective fault diagnosis of axle box bearing, by using condition-based maintenance (CBM) to replace the current widespread use of time-based maintenance and failure repair, not only can avoid train accidents, but also can reduce the operation cost of rail transport greatly. Fault diagnosis of the bearings based on vibration signal analysis is one of the basic fault diagnosis methods in the industry. During the past several decades, the Fourier spectrum analysis has been widely used in the field of signal analysis. However, there are some crucial restrictions to the use of Fourier transform. For example, the signal generated by the inspected machine must be linear and stationary; otherwise, the results of Fourier spectrum would have little physical sense. Unfortunately, the vibration signals generated by the train axle box bearing are often nonstationary and nonlinear. Therefore, Fourier transform cannot meet the requirements of fault diagnosis of the axle box bearings when the train is in operation.

To overcome the limitations of the Fourier transform, Huang proposed the empirical mode decomposition (EMD) method [1]. This method can effectively handle nonlinear and nonstationary signals by decomposing the signal into a series of single frequency components of the IMFs. Many researchers have used the EMD in nonlinear and nonstationary signal processing. In 2006, Yu and Junsheng [2] analyzed the vibration signal of rolling bearing in terms of the EMD energy entropy and calculated the IMF energy as the fault feature. In 2007, Yang et al. [3] analyzed IMF envelope spectrum and calculated the fault feature amplitude ratio and used support vector machine for fault classification. In 2010, Tang et al. [4] extracted the fault features of rolling bearings by combining morphology singular value decomposition with EMD. In 2012, Bin et al. [5] combined wavelet packet decomposition with EMD to make a contrast between IMF energy and IMF energy-torque and extracted IMF energytorque as the incipient fault feature of the rotating machinery. 
The majority of the literature takes the single type of feature parameters as a fault feature vector. In order to fully reflect the health condition of train axle box bearing, this paper proposes a fault diagnosis method based on multifeature parameters for train axle box bearings. In this diagnosis method, firstly, wavelet decomposition method is employed to eliminate noises in the vibration signals; then the EMD decomposition method on the denoised signals is employed for feature extraction. The bearing fault diagnosis system extracts 12 features, which include 5 statistical parameters in the time domain, 5 IMF energy-torque features, and 2 amplitude-ratio features. These 12 features can distinguish four types of state of a bearing, namely, normal bearing, outer ring fault, inner ring fault, and rolling body fault. In the end, a fault classifier based on BP neural network is realized to identify train axle box bearing fault automatically.

\section{Principle of EMD Algorithm}

The EMD algorithm proposed by Huang is employed to analyze the nonstationary signals by gradually partitioning the signal in different scale fluctuations or trends to produce a series of single frequency components of IMFs. The EMD algorithm can better reflect the local characteristics of the signals [6] compared with the wavelet analysis method. Before using the EMD method to decompose a complex signal, the following assumptions will be made [1]: the signal consists of a series of IMFs; each IMF has the same extreme points and zero-crossing point number; both of the envelope curves covering the local maxima and local minima have zero mean. EMD can be regarded as an adaptive filtering processing and the steps can be described as follows.

(1) Calculating the upper envelope line of the signal. For a given signal $x(t)$, determine all the local maximum values of the signal and then use the order spline interpolation method three times for fitting into the upper envelope line of the signal.

(2) Calculating the lower envelope line of the signal. Determine all the local minimum values of the signal and then use the order spline interpolation method three times to get the lower envelope line of the signal.

(3) Calculating the mean value $m_{1}(t)$ of the upper envelope line and the lower envelope line, with the original signal $x(t)$ being subtracted from the envelope mean value $m_{1}(t)$, we get the difference

$$
h_{1}(t)=x(t)-m_{1}(t) .
$$

(4) Determine whether $h_{1}(t)$ satisfies the IMF condition; if not, execute a second screening and take $h_{1}(t)$ as the new original data. Repeat step (1) to step (3); then the subtraction between $h_{1}(t)$ and its envelope mean value $m_{11}(t)$ is

$$
h_{11}(t)=h_{1}(t)-m_{11}(t) .
$$

If $h_{11}(t)$ still does not satisfy the IMF condition, then repeat the above process $k$ times until $h_{1 k}(t)$ can satisfy the condition. The first IMF of the signal $x(t)$ is denoted as $c_{1}(t)$ :

$$
c_{1}(t)=h_{1 k}(t) .
$$

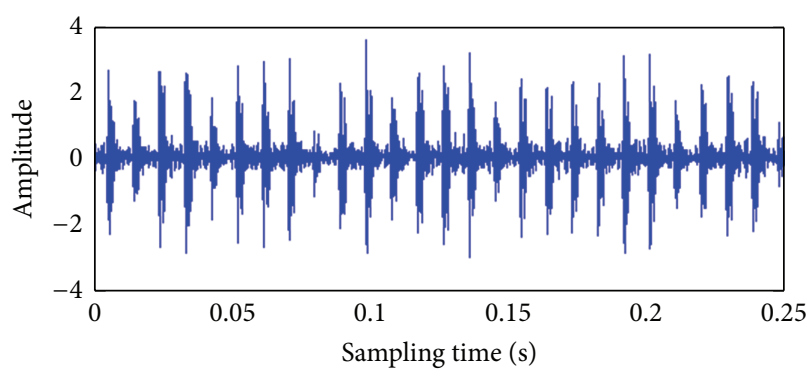

FIGURE 1: Original vibration signal.

(5) Separate $c_{1}(t)$ from the original signal $x(t)$; then the residual term $r_{1}(t)$ can be obtained:

$$
r_{1}(t)=x(t)-c_{1}(t) .
$$

Repeat the above process by taking the residual term $r_{1}(t)$ as the new original data until the residual term $r_{n}(t)$ is a monotonic function or less than a given value after $n$ times. At this time, the decomposition process of the original signal $x(t)$ is completed and can be expressed as

$$
x(t)=\sum_{i=1}^{n} c_{i}(t)+r_{n}(t) .
$$

By using the above method, we will get $\mathrm{n}$ IMFs and a residual term $r_{n}(t)$, which shows that the cycle length of the random signal is greater than the sampling frequency components and the cycle length is usually shown as a linear or slow changing trend error which is also known as the signal trends. It is often neglected in the subsequent analysis.

\section{The Analysis of Fault Signal Based on EMD}

Shown in Figure 1 is the original vibration signal of an axle box bearing with outer ring fault.

To extract the effective fault features of axle box bearings, the original vibration signal is denoised at the beginning. The actual signals and noised signals in the wavelet domain are of different properties and the wavelet coefficient amplitudes have different change trends with wavelet decomposition scales. With the wavelet decomposition scale increasing, the amplitude of the actual signal wavelet coefficient remains constant, while the amplitude of the noise signal wavelet coefficient will reduce to zero at a larger rate. Thus, wavelet decomposition method is a very effective signal denoising processing method. Up to date, several wavelet bases have been developed. Among them, the DB series, especially the DB4 wavelet basis, are widely used in the field of transient signal detection. The noises in fault detection signal from the train axle box are typical transient signals, so the DB4 wavelet basis is very effective in removing them. In this paper, 5-layer DB4 wavelet is employed to eliminate the noise. The denoised signal is shown in Figure 2.

By decomposing the denoised vibration signal with the EMD algorithm, 11 IMFs and 1 residue will be obtained. Because the EMD algorithm has the end effect, the decomposition will produce false IMF components. This paper adopts 
TABLE 1: The various IMFs and the correlation coefficients of the denoised vibration signals.

\begin{tabular}{lccccccccccc}
\hline IMF & $C_{1}$ & $C_{2}$ & $C_{3}$ & $C_{4}$ & $C_{5}$ & $C_{6}$ & $C_{7}$ & $C_{8}$ & $C_{9}$ & $C_{10}$ & $C_{11}$ \\
\hline Correlation coefficient & 0.140 & 0.179 & 0.191 & 0.335 & 0.521 & 0.513 & 0.310 & 0.172 & 0.079 & 0.081 & 0.042 \\
\hline
\end{tabular}

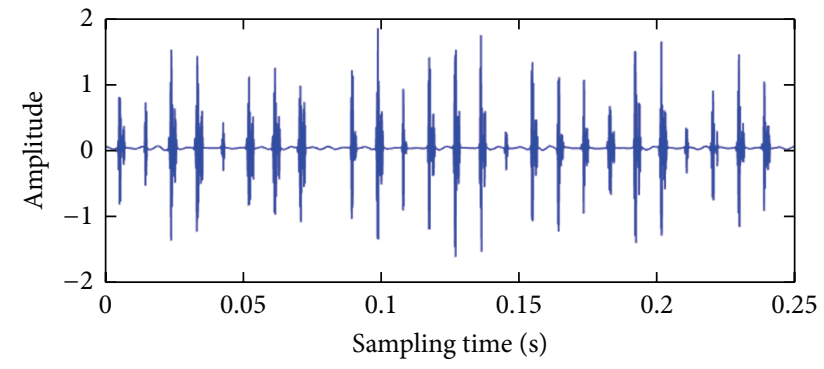

Figure 2: Denoised vibration signal.

the correlation coefficient method to eliminate the false IMF components [7]. Various IMFs and the correlation coefficient of the denoised vibration signal are shown in Table 1, from which it can be seen that the $c_{9}, c_{10}$, and $c_{11}$ of the IMFs are obviously false components and they should be incorporated into the residue. In this paper, the $8 \mathrm{IMFs}$ and 1 residue are selected by the correlation coefficient method, as shown in Figure 3, from which it can be seen that the 8 components all conform to the features of IMF.

\section{Fault Feature Extraction}

4.1. Feature Extraction Based on Time Domain Statistical Parameters. Time domain statistical parameters were divided into two types, dimensional and dimensionless. The shortcomings of the dimensional statistical parameters are that their values will change with the changes of the load, the speed, and other conditions. While the dimensionless statistical parameters do not have these shortcomings, this paper adapts five time domain dimensionless parameters; they are kurtosis value, crest factor, clearance factor, impulse factor, and shape factor. The calculations for these five factors are shown as follows.

Kurtosis value is as follows:

$$
K_{v}=\frac{\sum_{i=1}^{n} x_{i}^{4}}{n x_{\mathrm{rms}}^{4}}
$$

Crest factor is as follows:

$$
C_{f}=\frac{x_{\text {peak }}}{x_{\text {rms }}}
$$

Clearance factor is as follows:

$$
\mathrm{CL}_{f}=\frac{x_{\text {peak }}}{x_{r}}
$$

Impulse factor is as follows:

$$
I_{f}=\frac{x_{\text {peak }}}{|\bar{x}|} .
$$
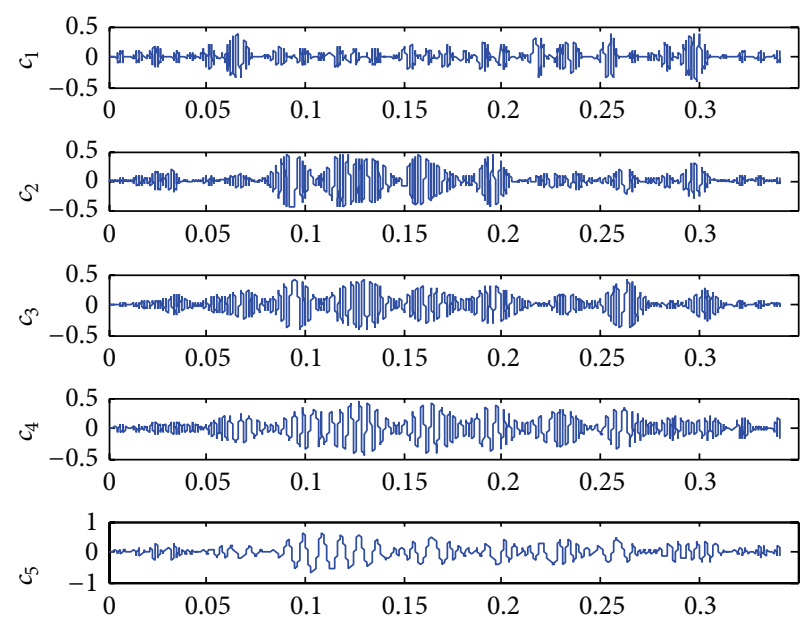

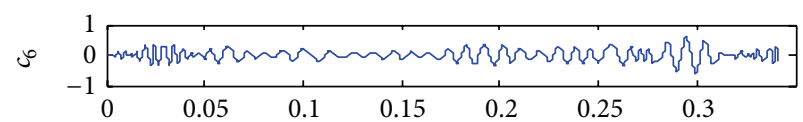

$5 \int_{-1}^{1} \underbrace{0}_{0}$
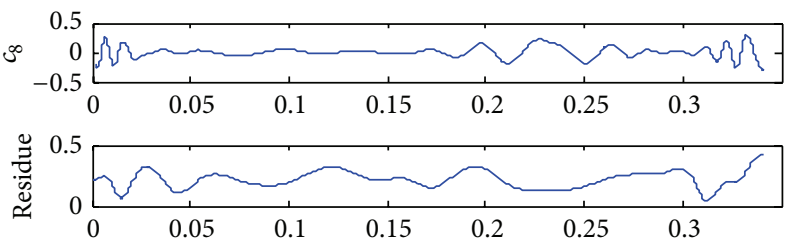

FIGURE 3: EMD decomposition results after being selected by correlation coefficient.

Shape factor is as follows:

$$
S_{f}=\frac{x_{\mathrm{rms}}}{|\bar{x}|},
$$

where $x_{\text {peak }}=\max \left\{\left|x_{i}\right|\right\}$ is the peak value; $x_{\text {rms }}=$ $\sqrt{(1 / n) \sum_{i=1}^{n} x_{i}^{2}}$ is root mean square value; $x_{r}=$ $\left((1 / n) \sum_{i=1}^{n} \sqrt{\left|x_{i}\right|}\right)^{2}$ is root square amplitude value; and $|\bar{x}|=(1 / n) \sum_{i=1}^{n}\left|x_{i}\right|$ is mean absolute magnitude.

Not only do these time domain feature parameters have higher sensitivities on the fault of axle box bearing, but also they have a stable corresponding relationship with the fault condition of an axle box bearing.

4.2. Feature Extraction Based on IMF Energy-Torque. The time scale and the energy distributed with the time scale are the two main parameters of a signal in the fault feature extraction. Compared with the vibration signal from normal bearing, the signal's energy of the vibration signal from fault bearing would be much different in the same frequency band. The energy of vibration signal coming from failure bearing 
will increase in a certain frequency band while the energy will decrease in another frequency band. Furthermore, different types of faults will show different distributions; therefore, the fault identification of an axle box bearing can be done according to the energy and the distribution regularity of various frequency components of the signal.

The vibration signal from an axle box bearing contains many nonlinear and nonstationary components. Most feature extraction methods based on EMD are to decompose the signal into a series of IMF components. Although using the energy and the energy entropy to analyze IMF achieves a better effect, it ignores the time parameters and cannot better reflect the essential features of a signal. While the feature extraction method using energy-torque instead of energy and energy entropy and taking distribution characteristics of the IMF on a time axis can better describe the essential features of signal and has more advantages in fault feature extraction than the methods based on energy and energy entropy [5], the steps of IMF energy-torque calculation are as follows.

(1) First, apply a wavelet denoising treatment to the original vibration signal of axle box bearing to eliminate noise interference.

(2) Decompose the denoised vibration signal by using the EMD method; then $\operatorname{IMF} c_{i}(t)(i=1,2,3, \ldots, n)$ will be obtained.

(3) Choose the proper IMF components and calculate energy-torque from the decomposition results of step (2); the IMF energy-torque can be calculated as

$$
E_{i}=\int_{-\infty}^{+\infty} t\left|c_{i}(t)\right|^{2} d t
$$

For discrete signals, the formula to calculate the energytorque is

$$
E_{i}=\sum_{k=1}^{m}(k \cdot \Delta t)\left|c_{i}(k \cdot \Delta t)\right|^{2}
$$

in which $m$ is the total number of sampling points, $k$ is sampling points, and $\Delta t$ is sampling period. For each chosen IMF, the energy-torques, $E_{1}, E_{2}, \ldots$, can be calculated from formula (12).

(4) Construct the feature vector $T$ in the elements of the energy-torque:

$$
T=\left[\begin{array}{llll}
E_{1} & E_{2} & \cdots & E_{n}
\end{array}\right] .
$$

When the energy-torques are larger numerical, normalize $T$ to get the normalized feature vector $T^{\prime}$ :

$$
T^{\prime}=\left[\begin{array}{llll}
\frac{E_{1}}{E} & \frac{E_{2}}{E} & \cdots & \frac{E_{n}}{E}
\end{array}\right]
$$

where

$$
E=\left(\sum_{i=1}^{n}\left|E_{i}\right|^{2}\right)^{1 / 2} .
$$

The formula for calculating IMF energy-torque is

$$
E_{i}=\int_{-\infty}^{+\infty}\left|c_{i}(t)\right|^{2} d t
$$

Comparing the calculation formula of IMF energy with that of IMF energy-torque, it can be seen that the energytorque considers the size of energy and the distribution status of the energy with time parameters, so it can better reveal the essential features of nonlinear and nonstationary signals.

The fault information of an axle box bearing mainly lies in the high frequency band, thus selecting the first 5 IMF components to calculate its energy-torque from the decomposition results of EMD.

4.3. Feature Extraction Based on IMF Envelope Spectrum. Vibration signals of axle box bearing usually show modulation characteristics. When the fatigue spalling failure of axle box bearing occurs, it will generate low frequencies periodic impact impulse signals. At the same time, the low frequencies periodic impact will stimulate the bearing's high frequency inherent vibration and exhibit modulation phenomenon. The regular envelope lines of the modulated wave can be employed to represent the low frequencies impact impulse signals generated by the fatigue spalling failure bearing, so the fault information is usually contained in the envelope of the signal. The signal envelope reflects the impact and the severity of each rotation cycle.

The EMD method can be seen as an adaptive filter whose bandwidth and center frequency change with the signal itself. Each IMF obtained by EMD is a single component modulation signal. These characteristics can be used to separate the modulation components produced by the axle box bearing with failure [8]. It can effectively extract the features of the original signal by this way.

Hilbert transform is a very efficient signal demodulation method. In this paper, the Hilbert transform is adopted to calculate the envelope signal of high frequency IMF component; the formula is

$$
H\left[c_{i}(t)\right]=\frac{1}{\pi} \int_{-\infty}^{+\infty} \frac{c_{i}(\tau)}{t-\tau} d \tau .
$$

After (17), the analytic signal of IMFs can be constructed as

$$
z_{i}(t)=c_{i}(t)+j H\left[c_{i}(t)\right]=a_{i}(t) e^{j \theta_{i}(t)},
$$

where the amplitude function $a_{i}(t)$ is

$$
a_{i}(t)=\sqrt{c_{i}^{2}(t)+H^{2}\left[c_{i}(t)\right]} .
$$

The amplitude functions $a_{i}(t)$ are the IMF envelope signals which are needed by the later analysis process.

The phase function $\theta_{i}(t)$ is

$$
\theta_{i}(t)=\arctan \frac{H\left[c_{i}(t)\right]}{c_{i}(t)} .
$$

According to formulas (18) to (20), each IMF component can be expressed as

$$
c_{i}(t)=a_{i}(t) \cos \left[\theta_{i}(t)\right] .
$$

It can be seen from (21) that each IMF component is a single component modulation signal which can be amplitude or frequency modulation. 


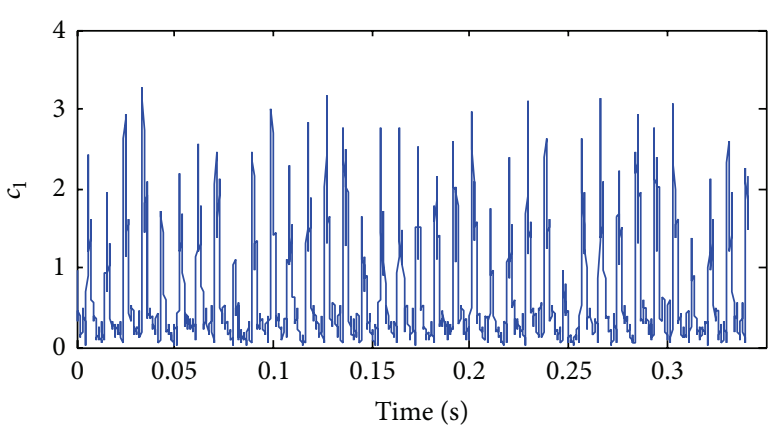

FIGURE 4: IMF signal envelope.

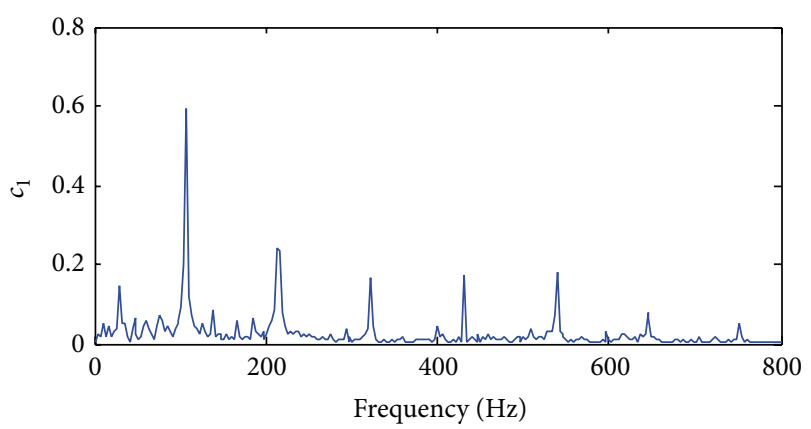

FIGURE 5: The IMF envelope spectrum.

According to the knowledge of rolling bearing, when a bearing with outer ring fault is running, a certain peak value (the peak value is called bearing fault frequency in the bearing industry) will appear in the frequency spectrum of the vibration signals from the failure bearing. The calculation formula for the certain peak value can be calculated [9]:

$$
F_{\text {ord }}=\frac{n}{2} f_{r}\left(1-\frac{B d}{P d} \cos \phi\right)
$$

where $f_{r}$ is the relative rotation frequency between inner circle and outer ring, $n$ is the number of the rolling elements, $B d$ is the roller elements' diameter, $P d$ is the pitch diameter of axle box bearing, and $\phi$ is the contact angle. The parameters of the bearing tested in this paper, respectively, are as follows: pitch diameter $P d=39.04 \mathrm{~mm}$, the diameter of rolling element $B d=7.94 \mathrm{~mm}$, the number of the rolling elements $n=9$, contact angle $\phi=0$, and rotation frequency $f_{r}=$ $29.83 \mathrm{~Hz}$. Taking these parameters into formula (22), we will get the fault frequency of outer ring fault axle box bearing $F_{\text {ord }}=106.93 \mathrm{~Hz}$.

The envelope of the first IMF of a vibration signal from an axle box bearing with outer ring failure is shown in Figure 4 . The envelope signal is obtained by the Hilbert transform demodulation method described as expressions (17) to (19).

Apply fast Fourier transform to the envelope signal shown in Figure 4, and obtain the IMF envelope spectrum, shown in Figure 5, from which it can be seen that the envelope spectrum of IMF component shows obviously the peak value $106.96 \mathrm{~Hz}$ which corresponds to the feature frequency of outer ring fault and its harmonics.
Similarly, the IMF envelope spectrum analysis method can also be used to recognize the failures which appear on the inner circle, rolling body of the axle box bearing. For normal axle box bearing, the envelope spectrum of IMF component has obvious peaks only at the corresponding rotation frequency and has no obvious peak at other frequencies.

Based on the envelope spectrum of IMF component, two very effective fault features named amplitude ratios are proposed [3]. They are defined as the ratio between the amplitude of the fault frequency of axle box bearing outer ring, inner circle fault frequency, and the rolling body fault frequency; these amplitude ratios are

$$
\begin{aligned}
& R_{1}=\frac{A\left(f_{\text {ord }}\right)}{A\left(f_{b d}\right)}, \\
& R_{2}=\frac{A\left(f_{\text {ird }}\right)}{A\left(f_{b d}\right)},
\end{aligned}
$$

where $A\left(f_{\text {ord }}\right)$ is the amplitude of outer ring fault frequency, $A\left(f_{\text {ird }}\right)$ is the amplitude of inner circle fault frequency, and $A\left(f_{b d}\right)$ is the amplitude of rolling body fault frequency.

\section{Fault Pattern Recognition}

Error backpropagation (BP) neural network has been widely used in the fault pattern recognition because of the advantages of association, memory, reasoning ability, nonlinear characteristics, and system parallel processing [10]. This paper adapts BP neural network as the fault expert system to identify the health status of an axle box bearing.

The fault diagnosis algorithm of an axle box bearing based on BP neural network has three processes, that is, construction of the network, training, and fault classification and identification. In this paper, 12 feature parameters belong to the time domain statistical parameters, IMF energy-torque, and feature amplitude ratios of the IMF envelope spectrum. The feature parameters are divided into two groups, one for the training of BP neural network and the other group for testing the classification capability of the trained BP neural network.

The fault diagnosis process of an axle box bearing based on BP neural network can be described as follows.

(1) Determining the input vector of the BP neural network. Extract 12 feature parameters as the fault feature vectors and arrange them as the input vectors of the BP neural network $X=\left[x_{1}, x_{2}, \ldots, x_{12}\right]^{T}$.

(2) Coding the fault types of axle box bearing. The outputs of BP neural network correspond to different fault types of the axle box bearing; the expected output for normal bearing is $(1,0,0,0)$, the expected output for outer ring fault bearing is $(0,1,0,0)$, the expected output for inner circle fault bearing is $(0,0,1,0)$, and the expected output for rolling body fault bearing is $(0,0,0,1)$.

(3) Determining the relative parameters of the BP neural network. The axle box bearing fault diagnosis system proposed in this paper adopts a three-layer BP neural network. The input feature vector is the 12 feature parameters of the axle box bearing and the network outputs are the four conditions 
TABLE 2: The node numbers of the hidden layer and the corresponding output errors.

\begin{tabular}{lcccccccccc}
\hline Node number & 8 & 9 & 10 & 11 & 12 & 13 & 14 & 15 & 16 & 17 \\
\hline Output error & 0.8806 & 0.7618 & 1.0974 & 0.5284 & 0.6335 & 0.6840 & 1.1221 & 1.4976 & 0.9287 & 0.8793 \\
\hline
\end{tabular}

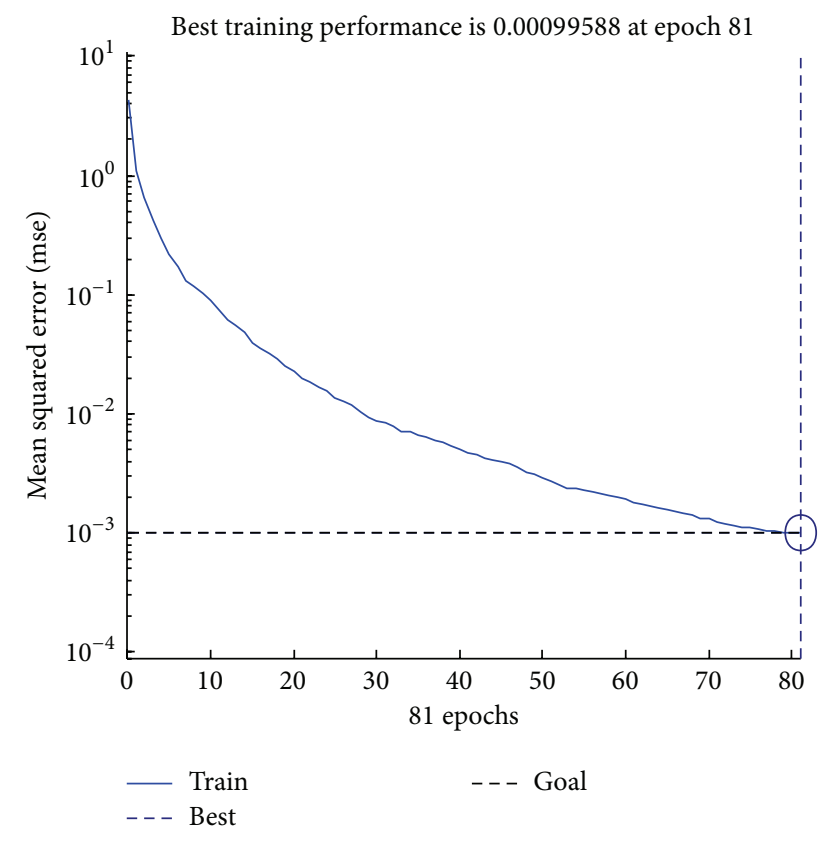

FIGURE 6: The error curve based on the multiple feature parameters of BP neural network.

of the axle box bearing. Each output contains 4 elements. So the node number of input layer is 12 ; the node number of output layer is 4 . If the node number of the hidden layer is too small, it is difficult to reach the training requirements; whereas if too much, it will increase the training time. In this paper, the following empirical formulas are adopted:

$$
\begin{gathered}
l=2 n+1, \\
l=\sqrt{n+m}+a .
\end{gathered}
$$

In (24), $l$ is the node number of the hidden layer neuron, $n$ is the node number of the input layer neuron, $m$ is the node number of the output layer neuron, and $a$ is a constant between 1 and 10 .

The empirical formula is only a rough estimation of the node number of the hidden layer. In practical application, an interval is set and the values are taken from the interval; then, by the trial and error method, the optimal node number of the hidden layer is determined. To avoid the impact of the node being too little or too much, the interval is set from 8 to 17. The corresponding network output error is shown in Table 2. It can be seen that when the BP neural network achieves smaller output error, the node number of the corresponding hidden layer is 11 .

In this paper, the linear transfer function purelin is selected as the output layer transfer function and the tangent type $\mathrm{S}$ transfer function tansig is selected as the hidden layer transfer function; it means that hidden layer outputs are real

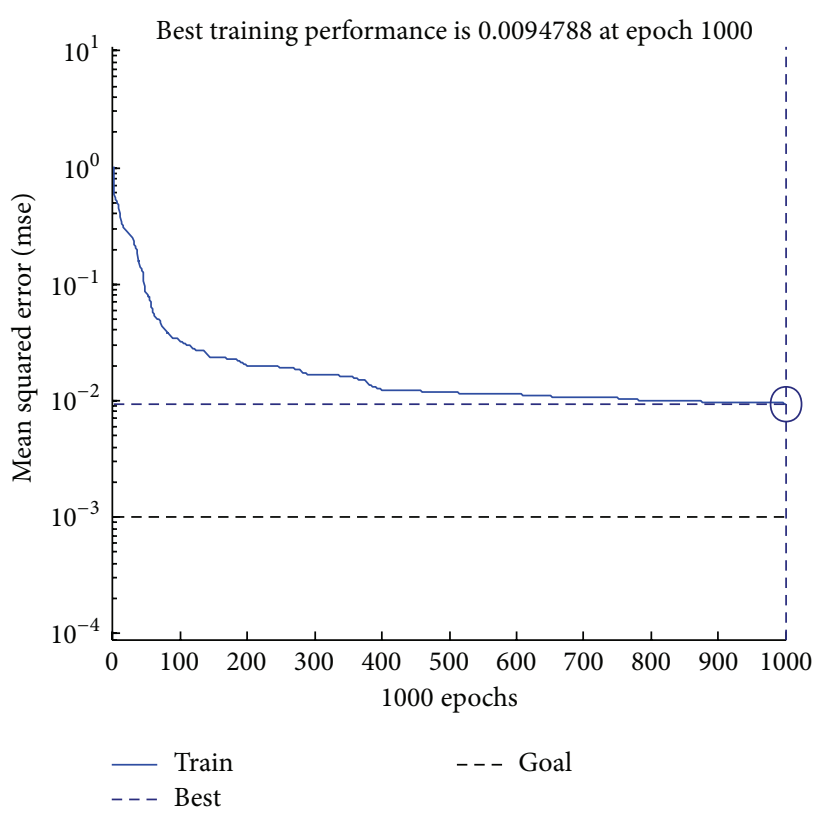

FIgURE 7: The error curve based on the time domain statistical parameters of BP neural network.

numbers from -1 to 1 . The trainscg is selected as the learning training function, which has the advantages of small memory space requirement and fast convergence rate. Also, when the training procedure does not converge, it will automatically stop training. The three network training parameters related to the conjugate gradient method are set as follows: the maximum number of training is set to 1000 , the learning rate is set to 0.05 , and the target error is set to 0.001 .

(4) The training of the BP neural network. Each of the four kinds of conditions of an axle box bearing has 20 groups of fault feature vectors, including normal bearing, outer ring fault, inner circle fault, and rolling body fault. Take 10 groups of samples to be used in training the BP neural network and the other 10 groups of samples as the tester. The training error curve of BP neural network is shown in Figure 6, and it can be seen that when the number of training reaches 81 times, the error reaches 0.001 and meets the requirements of precision. The training time lasts $1 \mathrm{~s}$.

(5) Fault recognition using BP neural network. For the four conditions of axle box bearing, the diagnosis results are shown in Table 3. For the four kinds of conditions of axle box bearing, the correct diagnosis rates achieve 100\%, 100\%, 90\%, and $90 \%$, respectively.

(6) Time domain statistical parameters as the input of BP neural network for fault recognition. Five time domain statistical parameters are adopted as the input vectors; the node number of the network input layer is 5 , the node number of the hidden layer is 11 , the node number of the output layer is 4 , and other parameters remain unchanged. Figure 7 is the 
TABLE 3: The results of BP neural network diagnosis.

\begin{tabular}{lcccc}
\hline Type & Normal & $\begin{array}{c}\text { Outer ring } \\
\text { fault }\end{array}$ & $\begin{array}{c}\text { Inner circle } \\
\text { fault }\end{array}$ & $\begin{array}{c}\text { Rolling } \\
\text { body fault }\end{array}$ \\
\hline $\begin{array}{l}\text { Correct } \\
\text { rates }\end{array}$ & $100 \%$ & $100 \%$ & $90 \%$ & $90 \%$ \\
\hline
\end{tabular}

TABLE 4: The diagnostic result based on the time domain statistical parameters of BP neural network diagnosis.

\begin{tabular}{lcccc}
\hline Type & Normal & $\begin{array}{c}\text { Outer circle } \\
\text { fault }\end{array}$ & $\begin{array}{c}\text { Inner circle } \\
\text { fault }\end{array}$ & $\begin{array}{c}\text { Rolling } \\
\text { body fault }\end{array}$ \\
\hline $\begin{array}{l}\text { Correct } \\
\text { rates }\end{array}$ & $100 \%$ & $90 \%$ & $90 \%$ & $90 \%$ \\
\hline
\end{tabular}

TABLE 5: The diagnostic result based on the IMF energy-torque of BP neural network diagnosis.

\begin{tabular}{lcccc}
\hline Type & Normal & $\begin{array}{c}\text { Outer circle } \\
\text { fault }\end{array}$ & $\begin{array}{c}\text { Inner circle } \\
\text { fault }\end{array}$ & $\begin{array}{c}\text { Rolling } \\
\text { body fault }\end{array}$ \\
\hline $\begin{array}{l}\text { Correct } \\
\text { rates }\end{array}$ & $100 \%$ & $90 \%$ & $90 \%$ & $100 \%$ \\
\hline
\end{tabular}

TABLE 6: The diagnostic result based on the feature amplitude ratio of IMF envelope spectrum of BP neural network diagnosis.

\begin{tabular}{lcccc}
\hline Type & Normal & $\begin{array}{c}\text { Outer circle } \\
\text { fault }\end{array}$ & $\begin{array}{c}\text { Inner circle } \\
\text { fault }\end{array}$ & $\begin{array}{c}\text { Rolling } \\
\text { body fault }\end{array}$ \\
\hline $\begin{array}{l}\text { Correct } \\
\text { rates }\end{array}$ & $80 \%$ & $100 \%$ & $80 \%$ & $90 \%$ \\
\hline
\end{tabular}

training error curve of the BP neural network. The error is only 0.009 when the maximum number of training reaches 1000; the training does not meet the error requirements and the process lasts 10 seconds. The change of the error is very small if the training continues; the diagnostic result of BP neural network is shown in Table 4. IMF energy-torque is given as the input vector of the BP neural network for fault recognition. Five IMF energy-torque feature parameters are adopted as the input vector of the BP neural network; the node number of the input layer is 5 , the node number of the hidden layer is 9 , the node number of the output layer is 4 , and other parameters remain unchanged. The training error curve is shown in Figure 8. It can be seen that the error reaches 0.001 when the number of training reaches 446 times in 5 seconds, and it meets the precision requirements. The fault recognition result of the BP neural network is shown in Table 5.

(7) The amplitude ratios of IMF envelope spectrum as the input vectors of BP neural network for classification. Two amplitude ratios of IMF envelope spectrum are adopted as the input features of BP neural network; the node number of the network input layer is 2 , the node number of the hidden layer is 6 , the node number of the output layer is 4 , and other parameters remain unchanged. The training error curve is shown in Figure 9; the error is only 0.087 when the maximum number of training reaches 1000 . It does not meet the error requirement and the process lasts 10 seconds. The variation of the error is too small to continue the training. The diagnostic result of BP neural network is shown in Table 6.

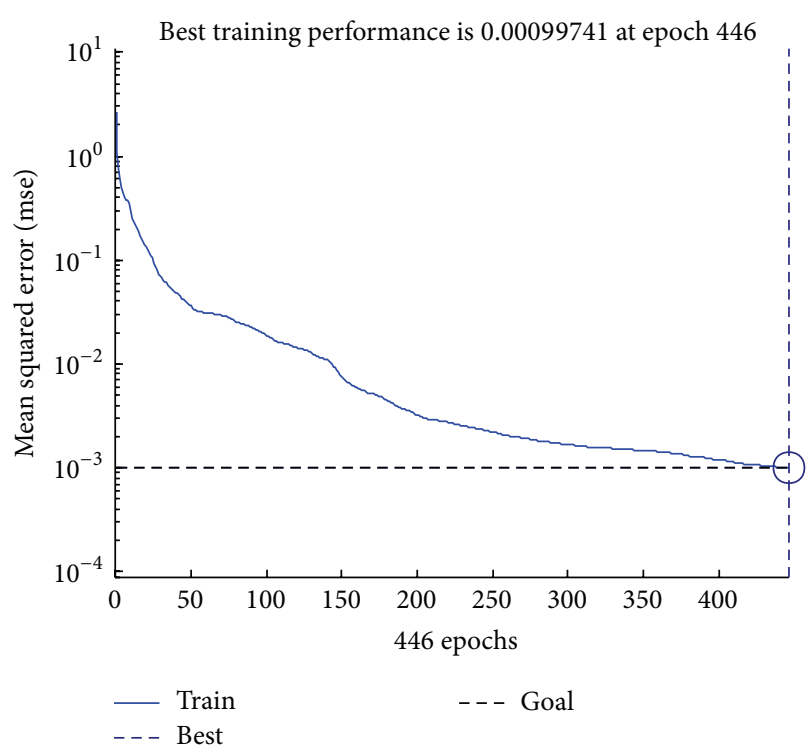

FIGURE 8: The error curve based on the IMF energy-torque of BP neural network.

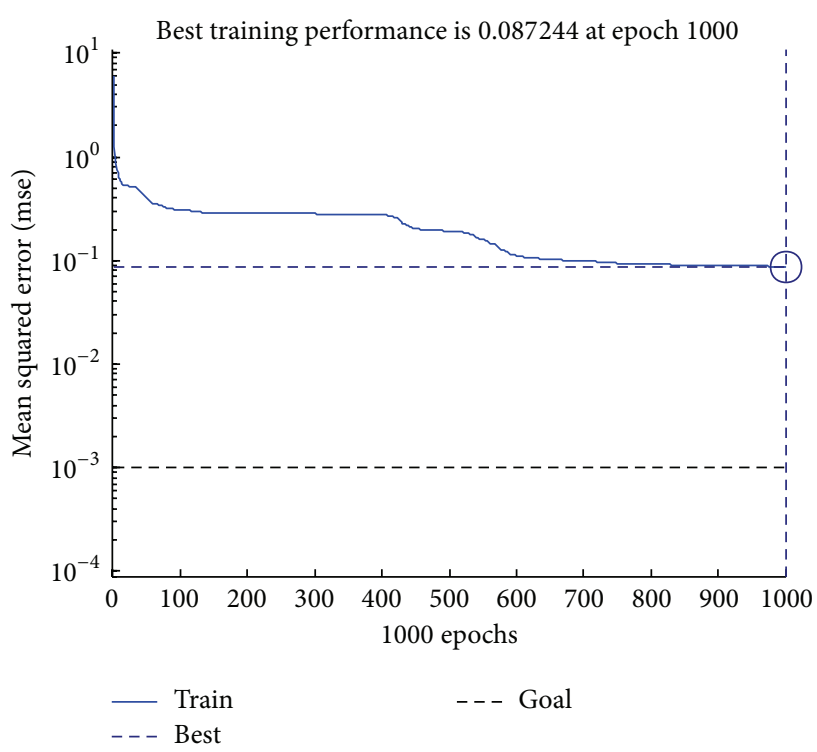

FIgURE 9: The error curve based on the feature amplitude ratio of IMF envelope spectrum of BP neural network.

(8) Result analysis. From the above results, the fault recognition of an axle box bearing has a higher accuracy based on the BP neural network. Taking the multiple feature parameters as the network input vectors, it will have faster convergence and higher precision. Only taking the time domain statistical parameters as the network input vectors, the convergence rate is slow and the precision is also low. Only taking the IMF energy-torque as the network input vectors, the convergence rate is slow but the accuracy can meet the requirements. Only taking the amplitude ratios features of IMF envelope spectrum as the network input vectors, it will have slow convergence and low accuracy. The training results are shown in Table 7. 
TABLE 7: Comparison of training results of BP neural network.

\begin{tabular}{lccc}
\hline The network input vector & $\begin{array}{c}\text { Training } \\
\text { error }\end{array}$ & $\begin{array}{c}\text { Training } \\
\text { time (s) }\end{array}$ & $\begin{array}{c}\text { Training } \\
\text { times }\end{array}$ \\
\hline $\begin{array}{l}\text { Multiple feature parameters } \\
\text { Time domain statistical }\end{array}$ & $10^{-3}$ & 1 & 81 \\
parameters & $10^{-2}$ & 10 & 1000 \\
$\begin{array}{l}\text { IMF energy-torque } \\
\begin{array}{l}\text { Feature amplitude ratios of } \\
\text { IMF envelope spectrum }\end{array}\end{array}$ & $10^{-3}$ & 5 & 446 \\
\hline
\end{tabular}

\section{Conclusions}

(1) The vibration signals of railway vehicle axle box bearings contain rich fault information. Aiming at vibration signals with nonlinear and nonstationary characteristics, a fault feature extraction method combining wavelet denoising and EMD is proposed. The EMD is based on adaptive decomposition of the signal and is suitable for dealing with nonlinear and nonstationary signals, and it is an effective feature extraction method.

(2) There are end effects in the EMD which will produce false IMF components. The false IMF components that contain no fault information can be removed by using the correlation coefficient method to calculate the IMFs and the correlation coefficients of the original signals.

(3) The fault diagnosis method proposed in this paper can achieve above $90 \%$ accuracy. The fault diagnosis system with multiple feature parameters as the input has better advantage both on convergence rate and on diagnosis accuracy.

\section{Conflict of Interests}

The authors declare that there is no conflict of interests regarding the publication of this paper.

\section{Acknowledgment}

This paper is supported by the National High-Tech Research and Development Program of China (2011AA110503).

\section{References}

[1] N. E. Huang, Z. Shen, S. R. Long et al., "The empirical mode decomposition and the Hilbert spectrum for nonlinear and non-stationary time series analysis," Proceedings of the Royal Society of London A, vol. 454, no. 1971, pp. 903-995, 1998.

[2] Y. Yu and C. Junsheng, "A roller bearing fault diagnosis method based on EMD energy entropy and ANN," Journal of Sound and Vibration, vol. 294, no. 1-2, pp. 269-277, 2006.

[3] Y. Yang, D. Yu, and J. Cheng, "A fault diagnosis approach for roller bearing based on IMF envelope spectrum and SVM," Measurement, vol. 40, no. 9-10, pp. 943-950, 2007.
[4] B. Tang, Y. Jiang, and X. Zhang, "Feature extraction method of rolling bearing fault based on singular value decompositionmorphology filter and empirical mode decomposition," Journal of Mechanical Engineering, vol. 46, no. 5, pp. 37-48, 2010.

[5] G. F. Bin, J. J. Gao, X. J. Li, and B. S. Dhillon, "Early fault diagnosis of rotating machinery based on wavelet packetsempirical mode decomposition feature extraction and neural network," Mechanical Systems and Signal Processing, vol. 27, no. 1, pp. 696-711, 2012.

[6] W. Yang and P. J. Tavner, "Empirical mode decomposition, an adaptive approach for interpreting shaft vibratory signals of large rotating machinery," Journal of Sound and Vibration, vol. 321, no. 3-5, pp. 1144-1170, 2009.

[7] Z. K. Peng, P. W. Tse, and F. L. Chu, "A comparison study of improved Hilbert-Huang transform and wavelet transform: application to fault diagnosis for rolling bearing," Mechanical Systems and Signal Processing, vol. 19, no. 5, pp. 974-988, 2005.

[8] W.-C. Tsao, Y.-F. Li, D. D. Le, and M.-C. Pan, "An insight concept to select appropriate IMFs for envelope analysis of bearing fault diagnosis," Measurement: Journal of the International Measurement Confederation, vol. 45, no. 6, pp. 1489-1498, 2012.

[9] SKF, Vibration Diagnostic Guide, 2009, http://www.skf.com/ portal/skf/home/aptitudexchange? contentId $=0.237932 .237933$ .237934 .237960 .867870 .

[10] C.-C. Wang, Y. Kang, P.-C. Shen, Y.-P. Chang, and Y.-L. Chung, "Applications of fault diagnosis in rotating machinery by using time series analysis with neural network," Expert Systems with Applications, vol. 37, no. 2, pp. 1696-1702, 2010. 


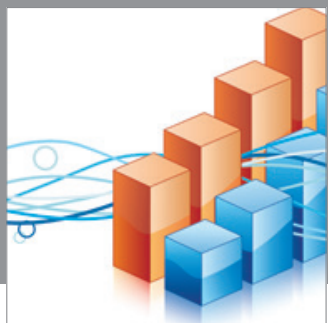

Advances in

Operations Research

mansans

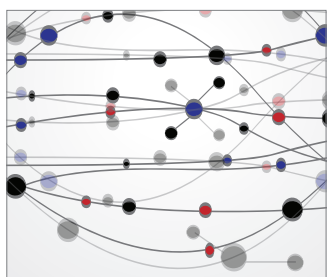

The Scientific World Journal
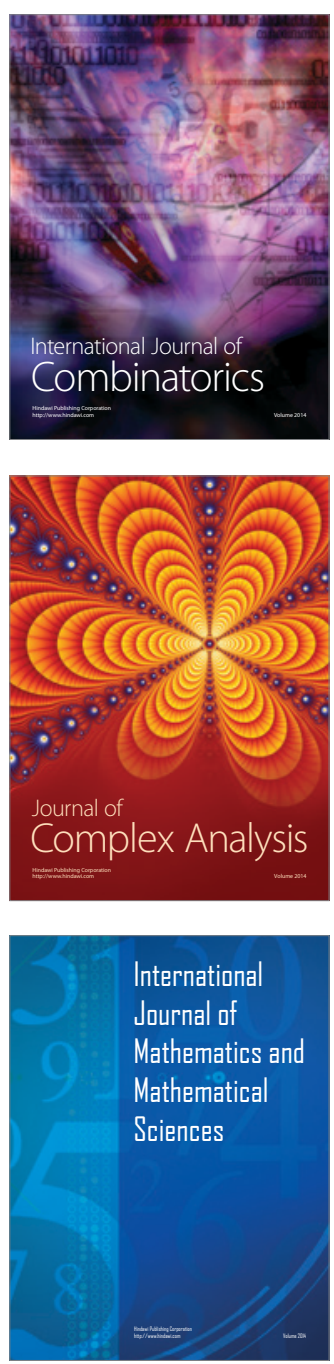
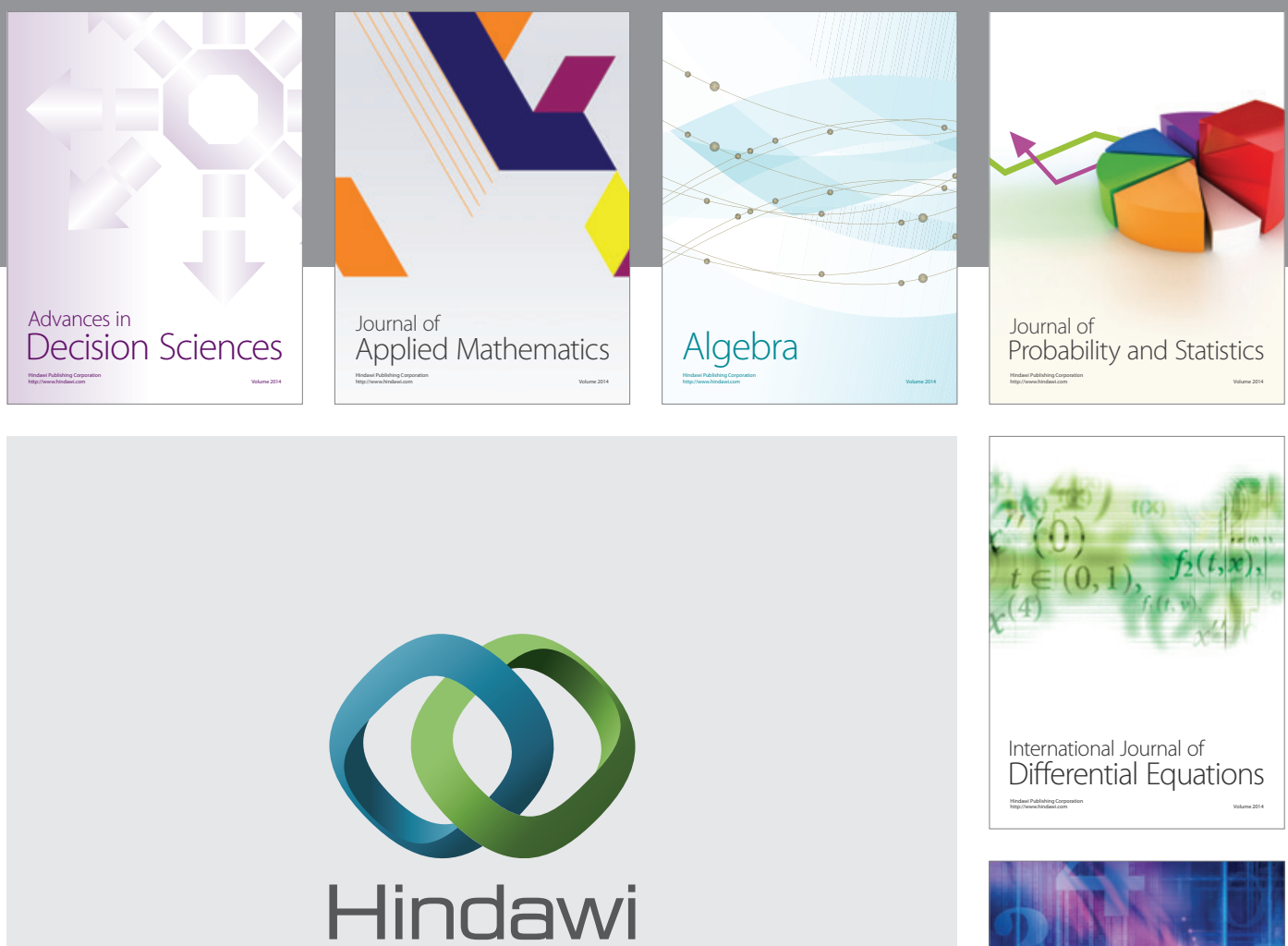

Submit your manuscripts at http://www.hindawi.com
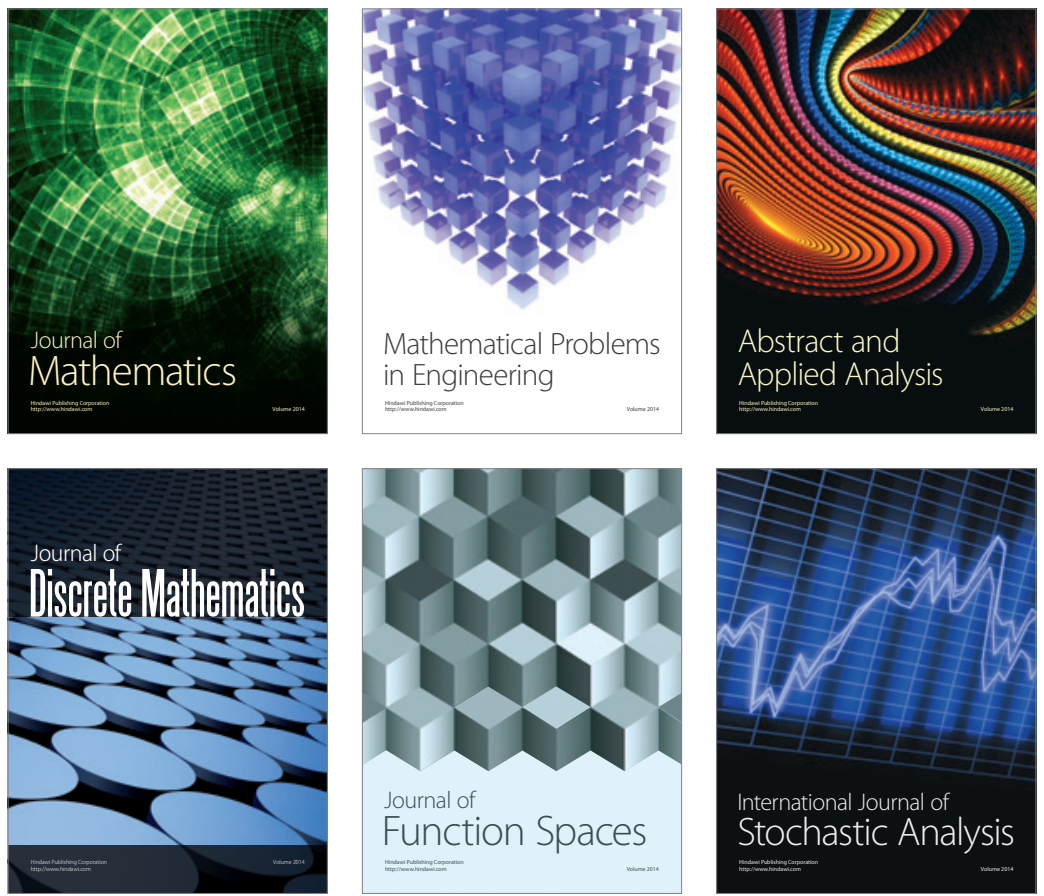

Journal of

Function Spaces

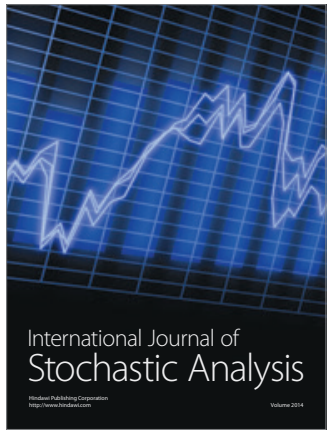

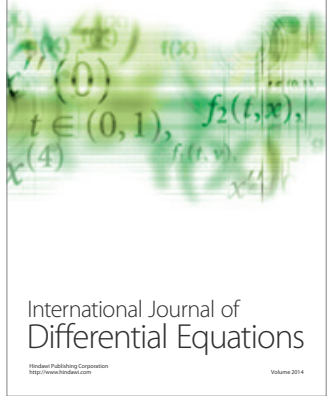
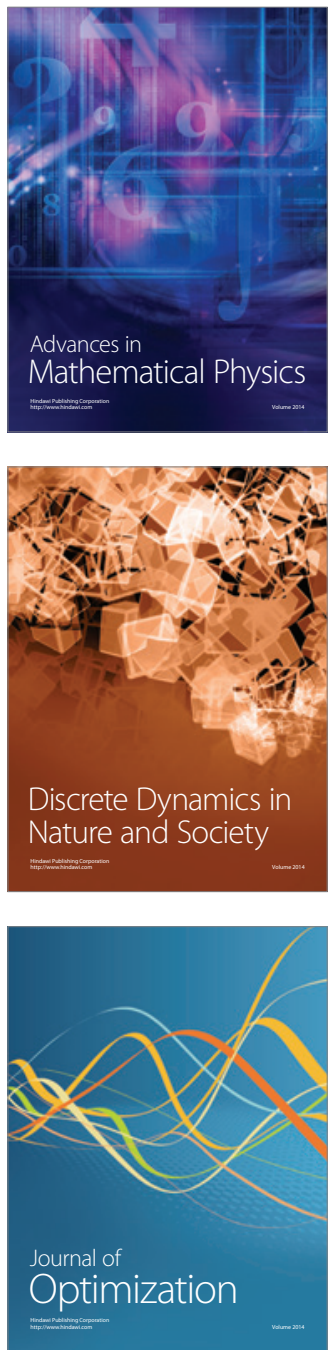